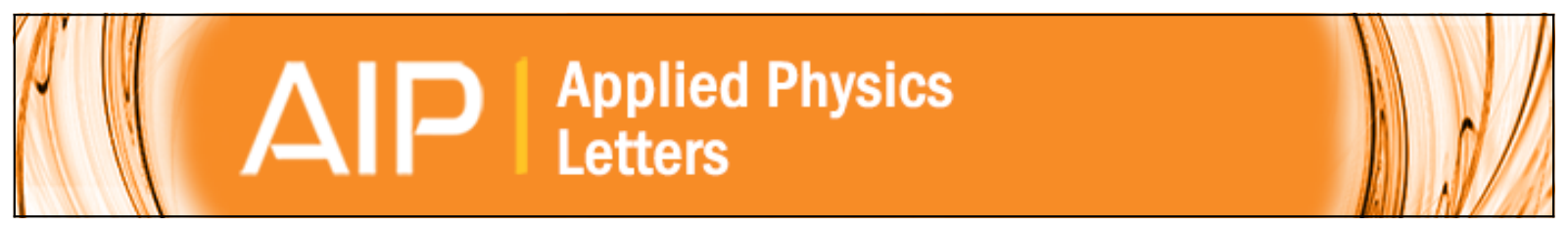

\title{
Monolithic vertical microcavities based on tetracene single crystals
}

Pompilio Del Carro, Andrea Camposeo, Luana Persano, Silvia Tavazzi, Marcello Campione, Antonio Papagni, Luisa Raimondo, Leonardo Silvestri, Peter Spearman, Roberto Cingolani, and Dario Pisignano

Citation: Applied Physics Letters 92, 063301 (2008); doi: 10.1063/1.2840153

View online: http://dx.doi.org/10.1063/1.2840153

View Table of Contents: http://scitation.aip.org/content/aip/journal/apl/92/6?ver=pdfcov

Published by the AIP Publishing

\section{Articles you may be interested in}

Optically pumped lasing from organic two-dimensional planar photonic crystal microcavity

Appl. Phys. Lett. 100, 213304 (2012); 10.1063/1.4720178

Polariton lasing in a hybrid bulk $\mathrm{ZnO}$ microcavity

Appl. Phys. Lett. 99, 161104 (2011); 10.1063/1.3650268

Monolithic single GaN nanowire laser with photonic crystal microcavity on silicon

Appl. Phys. Lett. 98, 021110 (2011); 10.1063/1.3540688

Vertical hybrid microcavity based on a polymer layer sandwiched between porous silicon photonic crystals Appl. Phys. Lett. 95, 163301 (2009); 10.1063/1.3245319

Continuously tunable laser emission from a wedge-shaped organic microcavity

Appl. Phys. Lett. 92, 163309 (2008); 10.1063/1.2913047 


\title{
Monolithic vertical microcavities based on tetracene single crystals
}

\author{
Pompilio Del Carro, ${ }^{1}$ Andrea Camposeo, ${ }^{1}$ Luana Persano, ${ }^{1}$ Silvia Tavazzi, ${ }^{2}$ \\ Marcello Campione, ${ }^{2}$ Antonio Papagni, ${ }^{2}$ Luisa Raimondo, ${ }^{2}$ Leonardo Silvestri, ${ }^{2}$ \\ Peter Spearman, ${ }^{2}$ Roberto Cingolani, ${ }^{1}$ and Dario Pisignano ${ }^{1, a)}$ \\ ${ }^{1}$ National Nanotechnology Laboratory (NNL) of INFM-CNR, c/o Distretto Tecnologico ISUFI, Università \\ del Salento, via Arnesano, I-73100 Lecce, Italy \\ ${ }_{2}^{2}$ Dipartimento di Scienza dei Materiali, Università degli Studi di Milano Bicocca, via Cozzi 53, I-20125 \\ Milano, Italy
}

(Received 9 October 2007; accepted 13 January 2008; published online 11 February 2008)

\begin{abstract}
The authors report on monolithic, light-emitting vertical microcavities based on an organic semiconductor single crystal. The devices are realized by reactive electron-beam deposition of dielectric mirrors and growth of tetracene crystals by physical vapor transport. The microcavities exhibit optical cavity modes in the visible range $(550-580 \mathrm{~nm})$ with full width at half maximum down to $2-3 \mathrm{~nm}$, corresponding to a $Q$ factor of about 200, and polarization-induced modal splitting up to $20 \mathrm{meV}$. These results open perspectives for the realization of polarized-emitting optoelectronic devices based on organic crystals. (c) 2008 American Institute of Physics.
\end{abstract}

[DOI: $10.1063 / 1.2840153$ ]

Low molar mass organic semiconductors in single crystal form exhibit unequalled performances in terms of carrier mobility and device reproducibility in organic electronics with respect to their amorphous counterpart. ${ }^{1}$ Among organic systems, polyacenes, such as tetracene (TCN), are particularly relevant because they form single crystals and can be sublimated on a variety of substrates in thin film form. ${ }^{2-8}$ Recently, this led to measuring room temperature charge carrier mobility up to $1.6 \mathrm{~cm}^{2} / \mathrm{V} \mathrm{s}$ in high-quality $\mathrm{TCN}$ single crystals, ${ }^{9}$ and to demonstrating organic light-emitting transistors. ${ }^{10}$ The emission properties of TCN also attracted special interest because of the observation of superradiance, ${ }^{11,12}$ which is particularly favored in polyacenes due to the combined effect of the polarization of the lowest energy optical transition and the spatial molecular arrangement within the crystal. ${ }^{13}$ These findings make TCN very promising for optoelectronic applications. Furthermore, embedding these organic single crystals in periodic photonic structures would allow one to further strengthen light-matter interaction, to achieve high spectral selectivity through the quantization of the electromagnetic field, and possibly to tailor the emission spectrum by the resonances of confined optical modes and optical transitions. In previous studies, for instance, polarization mode splitting was observed in vertical microcavities with inorganic materials, ${ }^{14-16}$ and amorphous organics, such as dye-doped ${ }^{17}$ and conjugated polymers. ${ }^{18,19}$ However, the implementation of specific, gentle nanofabrication procedures of photonic nanostructures and microcavities on organic semiconductor single crystals is a crucial prerequisite in order to develop light-emitting device architectures.

In this letter, we demonstrate monolithic vertical microcavities based on a TCN single crystal as active layer, clad by dielectric distributed Bragg reflectors (DBRs) realized by reactive electron-beam evaporation. The devices exhibit single cavity modes in the range of $550-580 \mathrm{~nm}$ with a full width at half maximum (FWHM) down to $2-3 \mathrm{~nm}$, corresponding to a $Q$ factor around 200. The investigation of the optical mode polarization leads to the observation of

\footnotetext{
${ }^{a)}$ Electronic mail: dario.pisignano@unile.it.
}

polarization-induced splitting up to $20 \mathrm{meV}$ at angles larger than $30^{\circ}$.

TCN was purchased by Sigma-Aldrich and purified by sublimation cycles. The purified powder was placed in a glass crucible and heated at $170{ }^{\circ} \mathrm{C}$ in a system for the crystal growth by the physical vapor transport method, using nitrogen as gas carrier. ${ }^{20}$ The crystallographic system of tetracene crystals is triclinic $(a=6.06 \AA, b=7.84 \AA, c$ $\left.=13.01 \AA, \alpha=77.1^{\circ}, \beta=72.1^{\circ}, \gamma=85.8^{\circ}, Z=2\right),{ }^{21}$ as schematized in the left inset of Fig. 1. The accessible crystal face is along the $a b$ plane and, typically, the crystal plates had lateral dimensions up to $2-3 \mathrm{~mm}$ and thickness up to $500 \mathrm{~nm}$, measured by a digital surface profilometer. The normal PL spectrum and the absorption spectrum taken at normal incidence on the $a b$ face and corresponding to the lower Davydov component clearly show vibronic progression peaks at 539 and $566 \mathrm{~nm}$, and at 442, 477, and $519 \mathrm{~nm}$, respectively

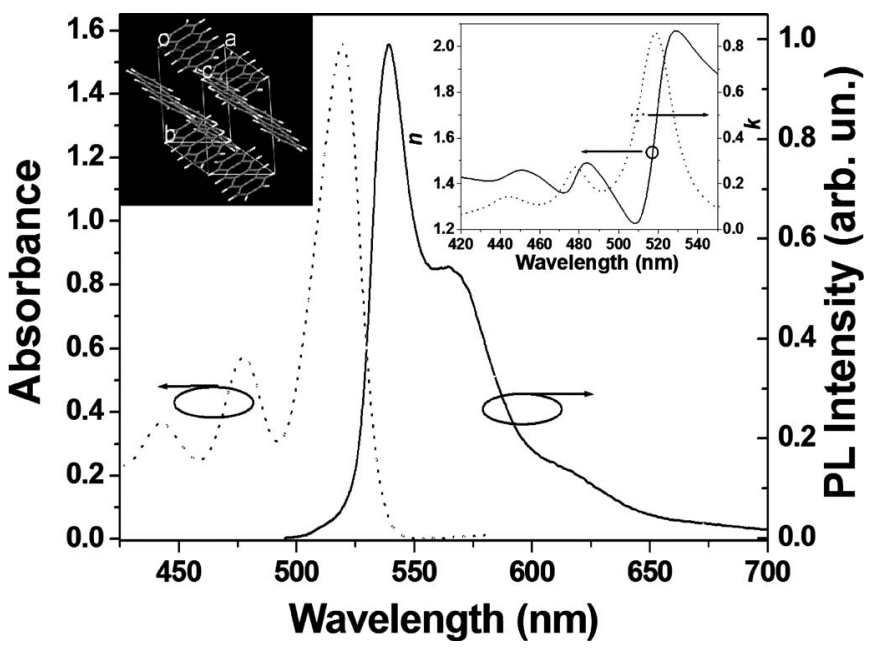

FIG. 1. Polarized absorption spectrum taken at normal incidence (dotted line, left vertical scale) and PL spectrum (solid line, right scale) of TCN single crystals. Left inset: arrangement of the TCN molecules in the unit cell as viewed from the $c^{*}$ axis. Right inset: measured wavelength behavior of the real ( $n$, solid line, left scale) and imaginary ( $k$, dotted line, right scale) parts of the crystal refractive index. 


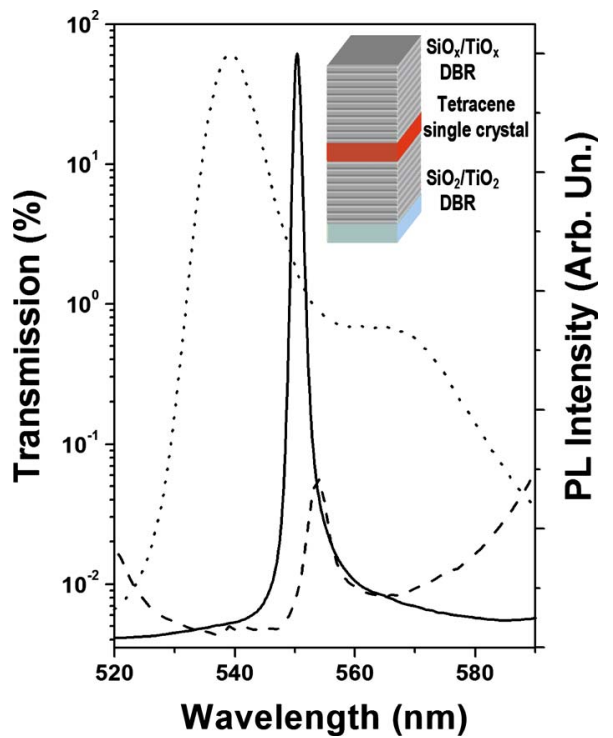

FIG. 2. (Color online) Microcavity transmission at normal incidence (dashed line, left vertical scale), and normal PL spectra from a microcavity (continuous line, right scale) and from a TCN crystal on a bottom DBR (dotted line, right scale). Inset: scheme of the TCN microcavity structure.

(Fig. 1). The real part ( $n$ ) of the crystal refractive index corresponding to the lower Davydov component is about 2 in the wavelength region corresponding to the PL maximum (right inset of Fig. 1), with a negligible imaginary $(k)$ part, as determined by means of a VASE spectroscopic ellipsometer (J.A. Woollam Co., Inc.) equipped with autoretarder and Xe lamp. ${ }^{22}$ We focus on the polarization corresponding to the lower Davydov component ${ }^{22,23}$ because we selected this polarization of the incident light when measuring the emission spectra of the TCN crystal with and without the microcavity. By maximizing the absorbance at normal incidence, this direction of polarization was found to lie approximately along the $a$ axis, in agreement with the calculated direction obtained from dipole lattice sums. ${ }^{22}$ The scheme of the TCN-based microcavities is displayed in the inset of Fig. 2. The bottom DBR, deposited onto Corning glass substrates at $260{ }^{\circ} \mathrm{C}$, is composed by $8.5 \lambda / 4$ pairs of $\mathrm{SiO}_{2}$ (with thickness, $d_{1}=88-90 \mathrm{~nm}$ ) and $\mathrm{TiO}_{2}$ (with thickness, $d_{2}=56-59 \mathrm{~nm}$ ) layers as low- and high-refractive index mediums, respectively. For the realization of the microcavities, TCN crystals were directly grown on the surface of the bottom DBR, allowing all the subsequent sample handling and measurements without any appreciable loss of adhesion from the silica surface. The thickness of the grown crystals embedded in the devices was comprised between 270 and $450 \mathrm{~nm}$, roughly corresponding to the cavity length $L_{C}$. The top mirror, consisting of 10.5 periods of $67 \mathrm{~nm} \mathrm{TiO}_{x}$ and $95 \mathrm{~nm} \mathrm{SiO}$ layers, was subsequently evaporated on the TCN crystal at low temperature $\left(T<80^{\circ} \mathrm{C}\right)$, in order to avoid damage of the underlying crystal. ${ }^{19,24}$ The lower deposition temperature affects the refractive index of the resulting layer (2.00 instead of 2.20 and 1.41 instead of 1.44, respectively, for titania and silica at $550 \mathrm{~nm}),{ }^{24}$ and can consequently influence the microcavity losses. However, the refractive index contrast achieved between the compounds evaporated at low $T$ is large enough to fabricate broadband DBRs. In these experiments, the stop bands for the bottom and the top mirror were measured to be 200 and $150 \mathrm{~nm}$ wide, respectively, with reflectivity maxima larger than $99 \%$. The normal inci- dence transmittance through the mirrors and the microcavity was measured by a double-beam Cary 5000 spectrophotometer (Varian, Australia), whereas angle-resolved transmission measurements were performed by mounting the sample on a rotation holder, and focussing the light from a W lamp on a $500 \mu \mathrm{m}$ spot on the cavity surface. The cw-PL from the microcavity was measured by exciting the samples through the bottom DBR by a laser diode $(\lambda=405 \mathrm{~nm}$, Nichia, Japan) and collecting the emitted light using an optical fiber and a monochromator (iHR320, Jobin Yvon) equipped with a charge-coupled device (Simphony, Jobin Yvon). All the optical measurements were carried out in ambient atmosphere at room temperature.

Figure 2 presents a typical microcavity normal transmission spectrum, exhibiting a quality factor, $Q \approx \lambda / \Delta \lambda$, where $\Delta \lambda$ indicates the transmission peak FWHM $(2-3 \mathrm{~nm})$ for the cavity mode resonance at $\lambda$, in the range of 180-270. In order to excite the PL from TCN crystal in the microcavity, particular attention was paid to employ light polarized along the direction of maximum absorbance at normal incidence. We also checked that the bottom DBR of the monolithic microcavity is not able to induce appreciable changes in the direction of the excitation polarization. In Fig. 2, we also compare the PL spectrum of TCN in the microcavity with that of a crystal as deposited on the bottom DBR. The organic crystal exhibits a broad PL peaked at $539 \mathrm{~nm}$ with a linewidth of about $40 \mathrm{~nm}$, whereas the microcavity emission is peaked at the resonance wavelength $(550 \mathrm{~nm})$ and significantly narrowed, with a FWHM of $3 \mathrm{~nm}$, indicating that a spectral selection is induced by the resonator structure. These architectures can be applied within light-emitting diodes based on organic semiconductor crystals to enhance the luminance and to narrow the emission spectrum thus improving the color purity. In addition, by virtue of the polarizationmaintaining DBRs, these devices can be effectively modulated by all-optical gating, exploiting the dependence of the TCN absorption on the incident polarization, ${ }^{22}$ which can be controlled by external electro-optic modulators.

We studied the angular behavior of the cavity mode energy, finding an increase upon increasing the incidence angle $\Theta$ according to the dispersion relation, ${ }^{25}$

$$
E(\Theta)=E_{0}\left(1-\sin ^{2} \Theta / n_{\mathrm{eff}}^{2}\right)^{-1 / 2},
$$

where $n_{\text {eff }}$ and $E_{0}$ indicate the effective refractive index of the structure and the mode energy at normal direction, respectively. The results obtained for a microcavity with TCN thickness of $420 \mathrm{~nm}$ and an optical mode at $575 \mathrm{~nm}$ are shown in Fig. 3. For $\Theta>30^{\circ}$, the photon resonant mode splits in a twofold band, with an energy separation growing nonlinearly up to $20 \mathrm{meV}$. By polarized angle-resolved measurements, we found that the high-energy splitting peak is transversal-electric (TE) polarized and the low-energy one is transversal-magnetic (TM) polarized (inset of Fig. 3). The energies of the TM and TE resonance peaks shift from 2.15 to $2.45 \mathrm{eV}$ and to $2.47 \mathrm{eV}$, respectively, for $\Theta$ increasing from $0^{\circ}$ to $55^{\circ}$, and from the angular dependence of the two resonances, we extracted the effective refractive indexes of the modes according to Eq. (1), finding $n_{\text {eff }}=1.73$ and 1.69, respectively (fitting lines in Fig. 3). The cavity-mode frequency $\omega_{m}$ of the TCN-based device is roughly $\omega_{m}(\Theta)=\left[L_{c} \omega_{c}(\Theta)+L_{\mathrm{DBR}}(\Theta) \omega_{s}(\Theta)\right] / L_{\text {eff }}(\Theta)$, where $\omega_{c}$ $=2 \pi c / n_{c} L_{c} \cos \Theta_{c}$, with $\Theta_{c}=\arcsin \left(\sin \Theta / n_{c}\right)$, is the FabryPerot frequency, $L_{\mathrm{DBR}}$ is the penetration depth of light inside 


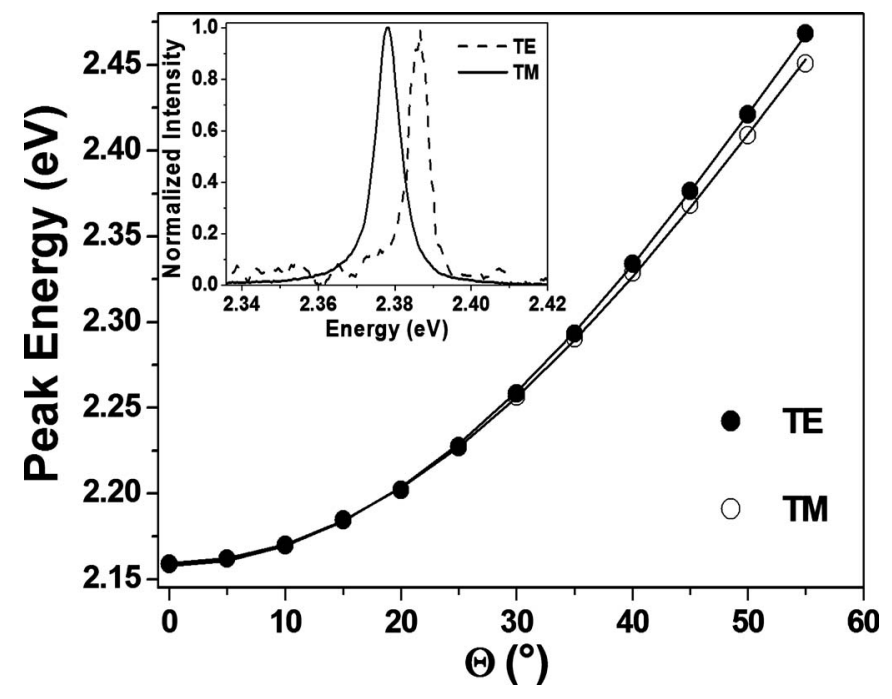

FIG. 3. Angular dependences of the resonance frequencies, extracted by transmission measurements, for TE (full dots) and TM (open circles) polarizations, for a TCN microcavity with crystal thickness $420 \mathrm{~nm}$. The superimposed continuous lines represent the fitting curves calculated according to Eq. (1). Inset: TE (dotted line) and TM (continuous line) transmission spectra for $\Theta=40^{\circ}$.

the DBR, $L_{\text {eff }}$ is the effective cavity length, and $\omega_{s}$ is the central frequency of the DBR stop band. For low refractive index contrasts, the polarization-induced splitting is controlled by the mismatch between $\omega_{s}$ and $\omega_{c}: \omega_{m}^{\mathrm{TM}}(\Theta)$ $-\omega_{m}^{\mathrm{TE}}(\Theta)=A\left[\omega_{s}(0)-\omega_{c}(0)\right] \sin ^{2}(\Theta)$, where $A$ is a constant. Consequently, for the TM mode to have lower energy with respect to the TE, one needs $\omega_{s}<\omega_{c}$ which is the case of the microcavity structure studied here, for which $\omega_{s}(0)=3.4 \pi c$ $\times 10^{-3}$ and $\omega_{c}(0)=3.5 \pi c \times 10^{-3} \mathrm{~nm}^{-1}$.

In summary, we fabricated monolithic, light-emitting vertical microcavities based on an organic crystal semiconductor. The device exhibited a $Q$ factor around 200, emission from the active layer, and polarization splitting up to $20 \mathrm{meV}$ at angles larger than $30^{\circ}$. The insertion of organic crystals in vertical microcavities extends the potential of this class of materials, opening the way towards the large scale integration of high-density light-emitting and modulation devices on chip.

The authors thank the support from the Italian Minister of Education, University and Research through the PRIN program and the Apulia Regional Explorative Project PE_086. L. R. and L. S. thank Sovvenzione Globale Ingenio for financial support given by Fondo Sociale Europeo, Ministero del Lavoro e della Previdenza Sociale, and Regione Lombardia.

${ }^{1}$ M. E. Gershenson, V. Podzorov, and A. F. Morpurgo, Rev. Mod. Phys. 78, 973 (2006).

${ }^{2}$ A. Wappelt, A. Bergmann, A. Napiwotzki, H. J. Eichler, H. J. Jupner, A. Kummrow, A. Lau, and S. Woggon, J. Appl. Phys. 78, 5192 (1995).

${ }^{3}$ F. Cicoira, C. Santato, F. Dinelli, M. Murgia, M. A. Loi, F. Biscarini, R. Zamboni, P. Heremans, and M. Muccini, Adv. Funct. Mater. 15, 375 (2005).

${ }^{4}$ A. L. Briseno, J. Aizenberg, Y. J. Han, R. A. Penkala, H. Moon, A. J. Lovinger, C. Kloc, and Z. Bao, J. Am. Chem. Soc. 127, 12164 (2005).

${ }^{5}$ B. Lu, H. J. Zhang, H. Huang, H. Y. Mao, Q. Chen, H. Y. Li, P. He, and S. N. Bao, Appl. Surf. Sci. 245, 208 (2005).

${ }^{6}$ C. Santato, I. Manunza, A. Bonfiglio, F. Cicoira, P. Cosseddu, R. Zamboni, and M. Muccini, Appl. Phys. Lett. 86, 141106 (2005).

${ }^{7}$ S. Soubatch, R. Temirov, M. Weinhold, and F. S. Tautz, Surf. Sci. 600, 4679 (2006).

${ }^{8}$ N. Moriguchi, T. Nishikawa, T. Anezaki, A. Unno, M. Tachibana, and K. Kojima, Physica B 376-377, 276 (2006).

${ }^{9}$ Y. Xia, V. Kalihari, C. D. Frisbie, N. K. Oh, and J. A. Rogers, Appl. Phys. Lett. 90, 162106 (2007).

${ }^{10}$ A. Hepp, H. Heil, W. Weise, M. Ahles, R. Schmechel, and H. von Seggern, Phys. Rev. Lett. 91, 157406 (2003).

${ }^{11}$ S.-H. Lim, T. G. Bjorklund, F. C. Spano, and C. J. Bardeen, Phys. Rev. Lett. 92, 107402 (2004).

${ }^{12}$ M. Voigt, A. Langner, P. Schouwink, J. M. Lupton, R. F. Mahrt, and M. Sokolowski, J. Chem. Phys. 127, 114705 (2007).

${ }^{13}$ F. C. Spano, J. Chem. Phys. 120, 7643 (2004).

${ }^{14}$ C. Y. Hu, H. Z. Zheng, J. D. Zhang, H. Zhang, F. H. Yang, and Y. P. Zeng, Appl. Phys. Lett. 82, 665 (2003).

${ }^{15}$ G. Panzarini, L. C. Andreani, A. Armitage, D. Baxter, M. S. Skolnick, V. N. Astratov, J. S. Roberts, A. V. Kavokin, M. R. Vladimrova, and M. A. Kaliteevsky, Phys. Rev. B 59, 5082 (1999).

${ }^{16}$ A. A. Dukin, N. A. Feoktistov, V. G. Golubev, A. V. Medvedev, A. B. Pevtsov, and A. V. Sel'kin, Phys. Rev. E 67, 046602 (2003).

${ }^{17}$ Q. Song, L. Liu, T. Ling, L. Xu, and W. Wang, Appl. Phys. Lett. 82, 2939 (2003).

${ }^{18}$ T. Virgili, D. G. Lidzey, D. D. C. Bradley, and S. Walker, Synth. Met. 116, 497 (2001)

${ }^{19}$ L. Persano, E. Mele, R. Cingolani, and D. Pisignano, Appl. Phys. Lett. 87, 031103 (2005).

${ }^{20}$ C. Kloc, P. G. Simpkins, T. Siegrist, and R. A. Laudise, J. Cryst. Growth 182, 416 (1997)

${ }^{21}$ D. Holmes, S. Kumaraswamy, A. J. Matzger, and K. P. C. Vollhardt, Chem.-Eur. J. 5, 3399 (1999).

${ }^{22}$ S. Tavazzi, L. Raimondo, L. Silvestri, P. Spearman, A. Camposeo, M. Polo, and D. Pisignano (unpublished).

${ }^{23}$ K. Mizuno, A. Matsui, and G. J. Sloan, J. Phys. Soc. Jpn. 53, 2799 (1984).

${ }^{24}$ L. Persano, R. Cingolani, and D. Pisignano, J. Vac. Sci. Technol. B 23, 1654 (2005).

${ }^{25}$ M. S. Skolnick, T. A. Fisher, and D. M. Whittaker, Semicond. Sci. Technol. 13, 645 (1998). 